\title{
Diffeomorphic Metric Mapping of Hybrid Diffusion Imaging based on BFOR Signal Basis
}

\author{
Jia $\mathrm{Du}^{1}$, A. Pasha Hosseinbor ${ }^{3,4}$, Moo K. Chung ${ }^{4,5}$, Barbara B. Bendlin ${ }^{6}$, Gaurav \\ Suryawanshi ${ }^{3}$, Andrew L. Alexander ${ }^{3,4}$, and Anqi Qiu ${ }^{1,2}$ \\ ${ }^{1}$ Department of Bioengineering, National University of Singapore \\ ${ }^{2}$ Clinical Imaging Research Center, National University of Singapore \\ ${ }^{3}$ Department of Medical Physics, University of Wisconsin-Madison \\ ${ }^{4}$ Waisman Laboratory for Brain Imaging and Behavior, University of Wisconsin-Madison \\ ${ }^{5}$ Biostatistics and Medical Informatics, University of Wisconsin-Madison \\ ${ }^{5}$ Department of Medicine, University of Wisconsin-Madison
}

\begin{abstract}
In this paper, we propose a large deformation diffeomorphic metric mapping algorithm to align multiple $b$-value diffusion weighted imaging (mDWI) data, specifically acquired via hybrid diffusion imaging (HYDI), denoted as LDDMM-HYDI. We adopt the work given in Hosseinbor et al. (2012) and represent the $q$-space diffusion signal with the Bessel Fourier orientation reconstruction (BFOR) signal basis. The BFOR framework provides the representation of $\mathrm{mDWI}$ in the $q$-space and thus reduces memory requirement. In addition, since the BFOR signal basis is orthonormal, the $\mathbf{L}^{2}$ norm that quantifies the differences in $q$-space signals of any two mDWI datasets can be easily computed as the sum of the squared differences in the BFOR expansion coefficients. In this work, we show that the reorientation of the $q$-space signal due to spatial transformation can be easily defined on the BFOR signal basis. We incorporate the BFOR signal basis into the LDDMM framework and derive the gradient descent algorithm for LDDMM-HYDI with explicit orientation optimization. Using real HYDI datasets, we show that it is important to consider the variation of mDWI reorientation due to a small change in diffeomorphic transformation in the LDDMM-HYDI optimization.
\end{abstract}

\section{Introduction}

In order to accurately reconstruct the diffusion signal and ensemble average propagator (EAP), a thorough exploration of $q$-space is needed, which requires multiple $b$-value diffusion weighted imaging (mDWI). MDWI can characterize more complex neural fiber geometries when compared to single $b$-value techniques like diffusion tensor imaging (DTI) or high angular resolution diffusion imaging (HARDI). Hybrid diffusion imaging (HYDI) [15] is a mDWI technique that samples the diffusion signal along concentric spherical shells in $q$-space, with the number of encoding directions increased with each shell to increase the angular resolution with the level of diffusion weighting. Originally, HYDI employed the fast Fourier transform (FFT) to reconstruct the EAP. However, the recent advent of analytical EAP reconstruction schemes, which obtain closed-form expressions of the EAP, obviate the use of the FFT in HYDI. One such technique successfully validated on HYDI datasets is Bessel Fourier orientation reconstruction (BFOR) [12]. MDWI techniques like HYDI, however, have not been widely used by 
clinicians and neuroscientists partially due to their relatively long acquisition times. In addition, there is a lack of fundamental image analysis tools, such as registration, that can fully utilize their information.

In the last decades, researchers have spent great efforts on developing registration algorithms to align diffusion tensors derived from DTI and orientation distribution functions (ODFs) derived from HARDI [e.g., [1|14|8]]. However, registration algorithms directly based on DWIs are few. The direct alignment of DWIs in $q$-space utilizes the full diffusion information, is independent of the choice of diffusion models and their reconstruction algorithms (e.g., tensor, ODF), and unifies the transformation to align the local diffusion profiles defined at each voxel of two brains [5|16]18]. Dhollander et al.[5] developed an algorithm that transforms the diffusion signals on a single shell of $q$-space and preserves anisotropic as well as isotropic volume fractions. Yap et.al [16] proposed to decompose the diffusion signals on a single shell of $q$-space into a series of weighted diffusion basis functions, reorient these functions independently based on a local affine transformation, and then recompose the reoriented functions to obtain the final transformed diffusion signals. This approach provides the representation of the diffusion signal and also explicitly models the isotropic component of the diffusion signals to avoid undesirable artifacts during the local affine transformation. Zhang et al. [18] developed a diffeomorphic registration algorithm for aligning DW signals on a single shell of $q$-space.

Only recently, Dhollander et al. [6] aligned DWIs on multiple shells of $q$-space by first estimating transformation using a multi-channel diffeomorphic mapping algorithm, in which generalized fractional anisotrophy (GFA) images computed from each shell were used as mapping objects, and then applying the transformation to DWIs in each shell using the DWI reorientation method in [5]. This approach neglected possible influences of the DWI reorientation on the optimization of the spatial transformation. Hsu et al. [13] generalized the large deformation diffeomorphic metric image mapping algorithm [2] to DWIs in multiple shells of $q$-space and considered the image domain and $q$-space as the spatial domain where the diffeomorphic transformation is applied to. The authors claimed that the reorientation of DWIs is no longer needed as the transformation also incorporates the deformation due to the shape differences in diffusion profiles in $q$-space. It is a robust registration approach with the explicit consideration of the large deformation in both the image domain and the $q$-space. However, its computational complexity and memory requirement are high.

In this paper, we propose a new large deformation diffeomorphic metric mapping (LDDMM) algorithm to align HYDI datasets, denoted as LDDMM-HYDI. In particular, we adopt the BFOR framework in representing the $q$-space signal. Unlike Hsu et al. [13], the BFOR signal basis provides the representation of the $q$-space signal and thus reduces memory requirement. In addition, since the BFOR signal basis is orthonormal, the $\mathbf{L}^{2}$ norm that quantifies the differences in $q$-space signals can be easily computed as the sum of the squared differences in the BFOR expansion coefficients. In this work, we will show that the reorientation of $q$-space signal due to spatial transformation can be easily defined on the BFOR signal basis. Unlike the work in [6], we will incorporate the BFOR signal basis into the LDDMM framework and derive the gradient descent algorithm for 
solving the LDDMM-HYDI variational problem with explicit orientation optimization. As shown below, the main contributions of this paper are:

1. to seek large deformation for aligning HYDI datasets based on the BFOR representation of mDWI.

2. to derive the rotation-based reorientation of the $q$-space signal via the BFOR signal basis. This is equivalent to applying Wigner matrix to the BFOR expansion coefficients, where Wigner matrix can be easily constructed by the rotation matrix (see Section 2.1.

3. to derive the gradient descent algorithm for the LDDMM-HYDI variational problem with the explicit orientation optimization. In particular, we provide a computationally efficient method for calculating the variation of Wigner matrix due to the small variation of the diffeomorphic transformation (see Section 2.47.

4. to show that the LDDMM-HYDI gradient descent algorithm does not involve the calculation of the BFOR signal bases and hence avoids the discretization in $q$-space.

\section{Methods}

According to the work in [12], the $q$-space diffusion signal, $S(\mathbf{x}, \mathbf{q})$, can be represented as

$$
S(\mathbf{x}, \mathbf{q})=\sum_{n=1}^{N_{b}} \sum_{j=1}^{N_{Y}} c_{n j}(\mathbf{x}) \Psi_{n j}(\mathbf{q})
$$

where $\mathbf{x}$ and $\mathbf{q}$ respectively denote the image domain and $q$-space. $\Psi_{n j}(\mathbf{q})$ is the $n j$-th BFOR signal basis with its corresponding coefficient, $c_{n j}(\mathbf{x})$, at $\mathbf{x} . \Psi_{n j}(\mathbf{q})$ is given as

$$
\Psi_{n j}(\mathbf{q})=j_{l(j)}\left(\frac{\alpha_{n l(j)}|\mathbf{q}|}{\tau}\right) Y_{j}\left(\frac{\mathbf{q}}{|\mathbf{q}|}\right) .
$$

Here, $\alpha_{n l}$ is the $n^{\text {th }}$ root of the $l^{\text {th }}$ order spherical Bessel (SB) function of the first kind $j_{l} . \tau$ is the radial distance in $q$-space at which the Bessel function goes to zero. $Y_{j}$ are the modified real and symmetric spherical harmonics (SH) bases as given in [11]. $N_{Y}=\frac{(L+1)(L+2)}{2}$ is the number of terms in the modified SH bases of truncation order $L$, while $N_{b}$ is the truncation order of radial basis. We refer readers to [12] for more details.

Using the fact that the BFOR signal basis is orthonormal, the $\mathbf{L}^{2}$-norm of $S(\mathbf{x}, \mathbf{q})$ can be easily written as

$$
\|S(\mathbf{x}, \mathbf{q})\|_{2}=\sqrt{\int_{\mathbf{x} \in \mathbb{R}^{3}} \int_{\mathbf{q} \in \mathbb{R}^{3}} S^{2}(\mathbf{x}, \mathbf{q}) d \mathbf{q} d \mathbf{x}}=\sqrt{\int_{\mathbf{x} \in \mathbb{R}^{3}} \sum_{n=1}^{N_{b}} \sum_{j=1}^{N_{Y}} c_{n j}(\mathbf{x})^{2} d \mathbf{x} .}
$$

\subsection{Rotation-Based Reorientation of $S(\mathrm{x}, \mathrm{q})$}

We now discuss the reorientation of $S(\mathbf{x}, \mathbf{q})$ when rotation transformation $R$ is applied. We assume that the diffusion profile in each shell of $q$-space remains in the same shell 
after the reorientation. However, its angular profile in each shell of $q$-space is transformed according to the rotation transformation. Hence, we define

$$
R S(\mathbf{x}, \mathbf{q})=S\left(\mathbf{x},|\mathbf{q}| R^{-1} \frac{\mathbf{q}}{|\mathbf{q}|}\right) .
$$

According to the BFOR representation of $S(\mathbf{x}, \mathbf{q})$ in Eq. (1), we thus have

$$
R S(\mathbf{x}, \mathbf{q})=\sum_{n=1}^{N_{b}} \sum_{j=1}^{N_{Y}} c_{n j}(\mathbf{x}) j_{l(j)}\left(\frac{\alpha_{n l(j)}|\mathbf{q}|}{\tau}\right) Y_{j}\left(R^{-1} \frac{\mathbf{q}}{|\mathbf{q}|}\right) .
$$

This indicates that the rotation reorientation of $\mathrm{mDWI}$ is equivalent to applying the rotation transformation to the real spherical harmonics, $Y_{j}$. According to the work in [10], the rotation of $Y_{j}$ can be achieved by the rotation of their corresponding coefficients, yielding

$$
R S(\mathbf{x}, \mathbf{q})=\sum_{n=1}^{N_{b}}\left(\sum_{j=1}^{N_{Y}}\left(\sum_{j^{\prime}=1}^{N_{Y}} M_{j j^{\prime}} c_{n j^{\prime}}(\mathbf{x})\right)\right) j_{l(j)}\left(\frac{\alpha_{n l(j)}|\mathbf{q}|}{\tau}\right) Y_{j}\left(\frac{\mathbf{q}}{|\mathbf{q}|}\right)
$$

where $M_{j j^{\prime}}$ is the $j j^{\prime}$ th element of Wigner matrix $M(R)$ constructed based on $R$ (see details in [10]). We can see that the same Wigner matrix is applied to $c_{n j}$ at a fixed $n$. For the sake of simplicity, we rewrite Eq. (4) in the matrix form, i.e.,

$$
R S(\mathbf{x}, \mathbf{q})=(\mathbf{M}(R) \mathbf{c}(\mathbf{x}))^{\top} \boldsymbol{\Psi}(\mathbf{q})
$$

where $\mathbf{M}$ is a sparse matrix with $N_{b}$ diagonal blocks of $M(R)$. c is a vector that concatenates coefficients $c_{n j^{\prime}}$ in the order such that at a fixed $n, c_{n j^{\prime}}$ corresponds to $M(R) . \Psi(\mathbf{q})$ concatenates the BFOR signal basis.

\subsection{Diffeomorphic Group Action on $S(\mathrm{x}, \mathrm{q})$}

We define an action of diffeomorphisms $\phi: \Omega \rightarrow \Omega$ on $S(\mathbf{x}, \mathbf{q})$, which takes into consideration of the reorientation in $q$-space as well as the transformation of the spatial volume in $\Omega$. Based on the rotation reorientation of $S(\mathbf{x}, \mathbf{q})$ in Eq. (4), for a given spatial location $\mathbf{x}$, the action of $\phi$ on $S(\mathbf{x}, \mathbf{q})$ can be defined as

$$
\begin{aligned}
\phi \cdot S(\mathbf{x}, \mathbf{q}) & =S\left(\phi^{-1}(\mathbf{x}), R_{\phi^{-1}(\mathbf{x})}^{-1} \mathbf{q}\right) \\
& =\left(\mathbf{M}\left(R_{\phi^{-1}(\mathbf{x})}\right) \mathbf{c}\left(\phi^{-1}(\mathbf{x})\right)\right)^{\top} \boldsymbol{\Psi}(\mathbf{q}),
\end{aligned}
$$

where $R_{\mathrm{x}}$ can be defined in a way similar to the finite strain scheme used in DTI registration [1]. That is, $R_{\mathbf{x}}=\left(D_{\mathbf{x}} \phi D_{\mathbf{x}}^{\top} \phi\right)^{-\frac{1}{2}} D_{\mathbf{x}} \phi$, where $D_{\mathbf{x}} \phi$ is the Jacobian matrix of $\phi$ at $\mathbf{x}$. For the remainder of this paper, we denote this as

$$
\phi \cdot S(\mathbf{x}, \mathbf{q})=\left(\left(\mathbf{M}\left(R_{\mathbf{x}}\right) \mathbf{c}(\mathbf{x})\right)^{\top}\right) \circ \phi^{-1}(\mathbf{x}) \boldsymbol{\Psi}(\mathbf{q})
$$

where $\circ$ indicates as the composition of diffeomorphisms. 


\subsection{Large Deformation Diffeomorphic Metric Mapping for HYDIs}

The previous sections equip us with an appropriate representation of HYDI mDWI and its diffeomorphic action. Now, we state a variational problem for mapping HYDIs from one subject to another. We define this problem in the "large deformation" setting of Grenander's group action approach for modeling shapes, that is, HYDI volumes are modeled by assuming that they can be generated from one to another via flows of diffeomorphisms $\phi_{t}$, which are solutions of ordinary differential equations $\dot{\phi}_{t}=v_{t}\left(\phi_{t}\right), t \in[0,1]$, starting from the identity map $\phi_{0}=$ Id. They are therefore characterized by time-dependent velocity vector fields $v_{t}, t \in[0,1]$. We define a metric distance between a target HYDI volume $S_{\text {targ }}$ and a template HYDI volume $S_{\text {temp }}$ as the minimal length of curves $\phi_{t} \cdot S_{\text {temp }}, t \in[0,1]$, in a shape space such that, at time $t=1, \phi_{1} \cdot S_{\text {temp }}=S_{\text {targ }}$. Lengths of such curves are computed as the integrated norm $\left\|v_{t}\right\|_{V}$ of the vector field generating the transformation, where $v_{t} \in V$, where $V$ is a reproducing kernel Hilbert space with kernel $k_{V}$ and norm $\|\cdot\|_{V}$. To ensure solutions are diffeomorphic, $V$ must be a space of smooth vector fields. Using the duality isometry in Hilbert spaces, one can equivalently express the lengths in terms of $m_{t}$, interpreted as momentum such that for each $u \in V,\left\langle m_{t}, u \circ \phi_{t}\right\rangle_{2}=\left\langle k_{V}^{-1} v_{t}, u\right\rangle_{2}$, where we let $\langle m, u\rangle_{2}$ denote the $\mathbf{L}^{2}$ inner product between $m$ and $u$, but also, with a slight abuse, the result of the natural pairing between $m$ and $v$ in cases where $m$ is singular (e.g., a measure). This identity is classically written as $\phi_{t}^{*} m_{t}=k_{V}^{-1} v_{t}$, where $\phi_{t}^{*}$ is referred to as the pullback operation on a vector measure, $m_{t}$. Using the identity $\left\|v_{t}\right\|_{V}^{2}=\left\langle k_{V}^{-1} v_{t}, v_{t}\right\rangle_{2}=\left\langle m_{t}, k_{V} m_{t}\right\rangle_{2}$ and the standard fact that energy-minimizing curves coincide with constant-speed lengthminimizing curves, one can obtain the metric distance between the template and target

volumes by minimizing $\int_{0}^{1}\left\langle m_{t}, k_{V} m_{t}\right\rangle_{2} d t$ such that $\phi_{1} \cdot S_{\text {temp }}=S_{\text {targ }}$ at time $t=1$. We associate this with the variational problem in the form of

$$
J\left(m_{t}\right)=\inf _{m_{t}: \dot{\phi}_{t}=k_{V} m_{t}\left(\phi_{t}\right), \phi_{0}=\text { Id }} \int_{0}^{1}\left\langle m_{t}, k_{V} m_{t}\right\rangle_{2} d t+\lambda E\left(\phi_{1} \cdot S_{\text {temp }}, S_{\text {targ }}\right),
$$

where $\lambda$ is a positive scalar. $E$ quantifies the difference between deformed template $\phi_{1} \cdot S_{\text {temp }}$ and target $S_{\text {targ. }}$. Based on Eq. (3) and (5), $E$ is expressed in the form of

$$
E=\int_{\mathbf{x} \in \Omega}\left\|\left(\mathbf{M}\left(R_{\mathbf{x}}\right) \mathbf{c}_{\mathrm{temp}}(\mathbf{x})\right) \circ \phi^{-1}(\mathbf{x})-\mathbf{c}_{\mathrm{targ}}(\mathbf{x})\right\|_{2}^{2} d \mathbf{x} .
$$

\subsection{Gradient of $J$ with respect to $m_{t}$}

We now solve the optimization problem in Eq. (6) via a gradient descent method. The gradient of $J$ with respect to $m_{t}$ can be computed via studying a variation $m_{t}^{\epsilon}=m_{t}+\epsilon \widetilde{m}_{t}$ on $J$ such that the derivative of $J$ with respect to $\epsilon$ is expressed in function of $\widetilde{m}_{t}$. According to the general LDDMM framework derived in [9], we directly give the expression of the gradient of $J$ with respect to $m_{t}$ as

$$
\nabla J\left(m_{t}\right)=2 m_{t}+\lambda \eta_{t},
$$

where

$$
\eta_{t}=\nabla_{\phi_{1}} E+\int_{t}^{1}\left[\partial_{\phi_{s}}\left(k_{V} m_{s}\right)\right]^{\top}\left(\eta_{s}+m_{s}\right) d s
$$


Eq. (9) can be solved backward given $\eta_{1}=\nabla_{\phi_{1}} E . \partial_{\phi_{s}}\left(k_{V} m_{s}\right)$ is the partial derivative of $k_{V} m_{s}$ with respect to $\phi_{s}$.

In the following, we discuss the computation of $\nabla_{\phi_{1}} E$. We consider a variation of $\phi_{1}$ as $\phi_{1}^{\epsilon}=\phi_{1}+\epsilon h$ and denote the corresponding variation in $\mathbf{M}\left(R_{\mathbf{x}}\right)$ as $\mathbf{M}\left(R_{\mathbf{x}}^{\epsilon}\right)$. Denote $\hat{\mathbf{c}}(\mathbf{x})=\mathbf{M}\left(R_{\mathbf{x}}\right) \mathbf{c}_{\text {temp }}(\mathbf{x})$ for the simplicity of notation. We have

$$
\begin{aligned}
\left.\frac{\partial E}{\partial \epsilon}\right|_{\epsilon=0} & =\left.\int_{\mathbf{x} \in \Omega} \frac{\partial\left\|\left(\mathbf{M}\left(R_{\mathbf{x}}^{\epsilon}\right) \mathbf{c}_{\text {temp }}(\mathbf{x})\right) \circ\left(\phi_{1}^{\epsilon}\right)^{-1}(\mathbf{x})-\mathbf{c}_{\operatorname{targ}}(\mathbf{x})\right\|_{2}^{2}}{\partial \epsilon}\right|_{\epsilon=0} d \mathbf{x} \\
& =\underbrace{2 \int_{\mathbf{x} \in \Omega}\left\langle\hat{\mathbf{c}}(\mathbf{x}) \circ \phi_{1}^{-1}-\mathbf{c}_{\operatorname{targ}}(\mathbf{x}),\left.\nabla_{\mathbf{x}}^{\top} \hat{\mathbf{c}}(\mathbf{x}) \circ \phi_{1}^{-1} \frac{\partial\left(\phi_{1}^{\epsilon}\right)^{-1}}{\partial \epsilon}\right|_{\epsilon=0}\right\rangle d \mathbf{x}}_{\operatorname{term}(\mathrm{A})} \\
& +\underbrace{2 \int_{\mathbf{x} \in \Omega}\left\langle\hat{\mathbf{c}}(\mathbf{x}) \circ \phi_{1}^{-1}-\mathbf{c}_{\operatorname{targ}}(\mathbf{x}),\left(\left.\frac{\partial \mathbf{M}\left(R_{\mathbf{x}}^{\epsilon}\right) \mathbf{c}_{\mathrm{temp}}(\mathbf{x})}{\partial \epsilon}\right|_{\epsilon=0}\right) \circ \phi_{1}^{-1}\right\rangle d \mathbf{x}}_{\operatorname{term}(\mathbf{B})} .
\end{aligned}
$$

As the calculation of Term (A) is straightforward, we directly give its expression, i.e.,

$\operatorname{Term}(\mathrm{A})=-2 \int_{\mathbf{x} \in \Omega}\left\langle\left(D_{\mathbf{x}} \phi_{1}\right)^{-\top} \nabla_{\mathbf{x}} \hat{\mathbf{c}}(\mathbf{x})\left(\hat{\mathbf{c}}(\mathbf{x})-\mathbf{c}_{\operatorname{targ}}\left(\phi_{1}(\mathbf{x})\right)\right) \operatorname{det}\left(D_{\mathbf{x}} \phi_{1}\right), h\right\rangle d \mathbf{x}$.

This term is similar to that in the scalar image mapping case. It seeks the optimal spatial transformation $\phi_{t}$ in the gradient direction of image $\hat{\mathbf{c}}(\mathbf{x})$ weighted by the difference between the template and target images.

The computation of Term (B) involves the differential of $\mathbf{M}\left(R_{\mathbf{x}}\right)$ with respect to rotation matrix $R_{\mathrm{x}}$ and the variation of $R_{\mathrm{x}}^{\epsilon}$ with respect to the small variation of $\phi_{1}^{\epsilon}$. Let's first compute the derivative of $\mathbf{M}\left(R_{\mathbf{x}}\right)$ with respect to rotation matrix $R_{\mathbf{x}}$. According to the work in [3], the analytical form of this derivative can be solved using the Euler angle representation of $R_{\mathrm{x}}$ but is relatively complex. Here, we consider Wigner matrix $\mathbf{M}\left(R_{\mathbf{x}}\right)$ and the coefficients of the BFOR signal basis $\mathbf{c}_{\text {temp }}(\mathbf{x})$ together, which leads to a simple numeric approach for computing the derivative of $\hat{\mathbf{c}}(\mathbf{x})=$ $\mathbf{M}\left(R_{\mathbf{x}}\right) \mathbf{c}_{\text {temp }}(\mathbf{x})$ with respect to rotation matrix $R_{\mathbf{x}}$, i.e., $\nabla_{R_{\mathbf{x}}} \hat{\mathbf{c}}(\mathbf{x})$. Assume $\tilde{R}_{\mathbf{x}}=$ $e^{\delta U} R$, where $\delta U=\left[\begin{array}{ccc}0 & -\delta \mu_{3} & \delta \mu_{2} \\ \delta \mu_{3} & 0 & -\delta \mu_{1} \\ -\delta \mu_{2} & \delta \mu_{1} & 0\end{array}\right]$ is a skew-symmetric matrix parameterized

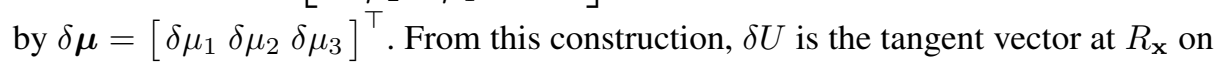
the manifold of rotation matrices and $\tilde{R}_{\mathrm{x}}$ is also a rotation matrix. Based on Taylor expansion, we have the first order approximation of $\mathbf{M}\left(\tilde{R}_{\mathbf{x}}\right) \mathbf{c}_{\text {temp }}(\mathbf{x})$ as

$$
\mathbf{M}\left(\tilde{R}_{\mathbf{x}}\right) \mathbf{c}_{\mathrm{temp}}(\mathbf{x}) \approx \hat{\mathbf{c}}(\mathbf{x})+\nabla_{R_{\mathbf{x}}}^{\top} \hat{\mathbf{c}}(\mathbf{x}) \delta \boldsymbol{\mu} .
$$

Hence, we can compute $\nabla_{R_{\mathbf{x}}} \hat{\mathbf{c}}(\mathbf{x})$ as follows. Assume $\delta U_{1}, \delta U_{2}, \delta U_{3}$ to be skewsymmetric matrices respectively constructed from $\left[\delta \mu_{1}, 0,0\right]^{\top},\left[0, \delta \mu_{2}, 0\right]^{\top},\left[0,0, \delta \mu_{3}\right]^{\top}$. 
We have

$$
\nabla_{R_{\mathbf{x}}} \hat{\mathbf{c}}(\mathbf{x}) \approx\left[\begin{array}{l}
\left(\mathbf{M}\left(e^{\delta U_{1}}\right) \hat{\mathbf{c}}(\mathbf{x})-\hat{\mathbf{c}}(\mathbf{x})\right)^{\top} / \delta \mu_{1} \\
\left(\mathbf{M}\left(e^{\delta U_{2}}\right) \hat{\mathbf{c}}(\mathbf{x})-\hat{\mathbf{c}}(\mathbf{x})\right)^{\top} / \delta \mu_{2} \\
\left(\mathbf{M}\left(e^{\delta U_{3}}\right) \hat{\mathbf{c}}(\mathbf{x})-\hat{\mathbf{c}}(\mathbf{x})\right)^{\top} / \delta \mu_{3}
\end{array}\right]
$$

It is worth noting that this formulation significantly reduces the computational cost for $\nabla_{R_{\mathbf{x}}} \hat{\mathbf{c}}(\mathbf{x})$. Since $\delta \boldsymbol{\mu}$ is independent of spatial location $\mathbf{x}, \mathbf{M}\left(e^{\delta U_{1}}\right), \mathbf{M}\left(e^{\delta U_{2}}\right)$, and $\mathbf{M}\left(e^{\delta U_{3}}\right)$ are only calculated once and applied to all $\mathbf{x}$.

We now compute the variation of $R_{\mathbf{x}}^{\epsilon}$ with respect to the small variation of $\phi_{1}^{\epsilon}$. This has been referred as exact finite-strain differential that was solved in [7] and applied to the DTI tensor-based registration in [17]. Here, we directly adopt the result from [17] and obtain

$$
\left.\frac{\partial R_{\mathbf{x}}^{\epsilon}}{\partial \epsilon}\right|_{\epsilon=0}=-F_{\mathbf{x}} \sum_{i=1}^{3}\left[\mathbf{r}_{i} \times\left(D_{\mathbf{x}} h^{\top}\right)_{i}\right]
$$

where $F_{\mathbf{x}}=-R_{\mathbf{x}}^{\top}\left(\operatorname{trace}\left(\left(D_{\mathbf{x}} \phi_{1} D_{\mathbf{x}}^{\top} \phi_{1}\right)^{1 / 2}\right) \operatorname{Id}-\left(D_{\mathbf{x}} \phi_{1} D_{\mathbf{x}}^{\top} \phi_{1}\right)^{1 / 2}\right)^{-1} R_{\mathbf{x}} \cdot \times$ denotes as the cross product of two vectors. $(A)_{i}$ denotes the $i$ th column of matrix $A \cdot \mathbf{r}_{i}=\left(R_{\mathbf{x}}^{\top}\right)_{i}$.

Given Eq. 12, and (13, we thus have

$$
\begin{aligned}
\operatorname{Term}(\mathrm{B}) & =-2 \int_{\mathbf{x} \in \Omega}\left\langle\hat{\mathbf{c}}(\mathbf{x}) \circ \phi_{1}^{-1}-\mathbf{c}_{\mathrm{targ}}(\mathbf{x}),\left(\nabla_{R_{\mathbf{x}}} \hat{\mathbf{c}}^{\top}(\mathbf{x}) F_{\mathbf{x}} \sum_{i=1}^{3}\left[\mathbf{r}_{i} \times\left(D_{\mathbf{x}} h^{\top}\right)_{i}\right]\right) \circ \phi_{1}^{-1}\right\rangle d \mathbf{x} \\
& =-2 \int_{\mathbf{x} \in \Omega} \boldsymbol{\omega}_{\mathbf{x}}^{\top} \sum_{i=1}^{3}\left[\mathbf{r}_{i} \times\left(D_{\mathbf{x}} h^{\top}\right)_{i}\right] d \mathbf{x} \\
& =-2 \int_{\mathbf{x} \in \Omega} \sum_{i=1}^{3}\left\langle\boldsymbol{\omega}_{\mathbf{x}} \times \mathbf{r}_{i}, \nabla_{\mathbf{x}} h_{i}\right\rangle d \mathbf{x}
\end{aligned}
$$

where

$$
\boldsymbol{\omega}_{\mathbf{x}}^{\top}=\left(\nabla_{R_{\mathbf{x}}} \hat{\mathbf{c}}\left(\hat{\mathbf{c}}(\mathbf{x})-\mathbf{c}_{\mathbf{t a r g}}\left(\phi_{1}(\mathbf{x})\right)\right)\right)^{\top} F_{\mathbf{x}} \operatorname{det}\left(D_{\mathbf{x}} \phi_{1}\right)
$$

and $h=\left[\begin{array}{lll}h_{1} & h_{2} & h_{3}\end{array}\right]^{\top} . D_{\mathbf{x}} h$ is approximated as

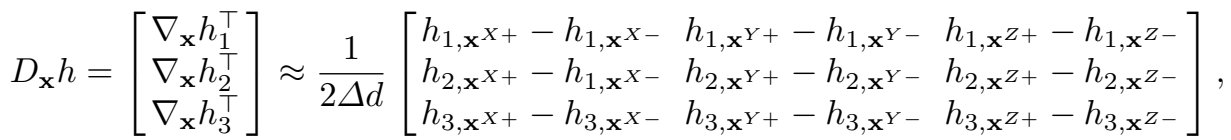

where $\left\{\mathbf{x}^{X+}, \mathbf{x}^{X-}, \mathbf{x}^{Y+}, \mathbf{x}^{Y+}, \mathbf{x}^{Z+}, \mathbf{x}^{Z-}\right\}$ are the neighbors of $\mathbf{x}$ in $x, y, z$ directions, respectively. $\Delta d$ is the distance of these neighbors to $\mathrm{x}$. Here, term (B) seeks the spatial transformation $\phi_{t}$ such that the local diffusion profiles of the template and target HYDIs have to be aligned. 
In summary, we have

$$
\begin{aligned}
\left.\frac{\partial E}{\partial \epsilon}\right|_{\epsilon=0} \approx-2 \int_{\mathbf{x} \in \Omega}\left\langle\left(D_{\mathbf{x}} \phi_{1}\right)^{-\top} \nabla_{\mathbf{x}} \hat{\mathbf{c}}(\mathbf{x})\left(\hat{\mathbf{c}}(\mathbf{x})-\mathbf{c}_{\operatorname{targ}}\left(\phi_{1}(\mathbf{x})\right)\right) \operatorname{det}\left(D_{\mathbf{x}} \phi_{1}\right), h\right\rangle d \mathbf{x} \\
-\frac{1}{\Delta d} \int_{\mathbf{x} \in \Omega} \sum_{k=1}^{3}\left\{\left\langle\boldsymbol{\omega}_{\mathbf{x}} \times \mathbf{r}_{k},\left[\begin{array}{c}
h_{k, \mathbf{x}^{X+}} \\
h_{k, \mathbf{x}^{Y+}} \\
h_{k, \mathbf{x}^{Z+}}
\end{array}\right]\right\rangle-\left\langle\boldsymbol{\omega}_{\mathbf{x}} \times \mathbf{r}_{k},\left[\begin{array}{c}
h_{k, \mathbf{x}^{X-}} \\
h_{k, \mathbf{x}^{Y-}} \\
h_{k, \mathbf{x}^{Z-}}
\end{array}\right]\right\rangle\right\} d \mathbf{x}
\end{aligned}
$$

Therefore, $\nabla_{\phi_{1}} E$ can be obtained from Eq. (16).

\subsection{Numerical Implementation}

We so far derive $J$ and its gradient $\nabla J\left(m_{t}\right)$ in the continuous setting. In this section, we elaborate the numerical implementation of our algorithm under the discrete setting. Since HYDI DW signals were represented using the orthonormal BFOR signal bases, both the computation of $J$ in Eq. (6) and the gradient computation in Eq. (16) do not explicitly involve the calculation $\boldsymbol{\Psi}(\mathbf{q})$. Hence, we do not need to discretize the $q$-space. In the discretization of the image domain, we first represent the ambient space, $\Omega$, using a finite number of points on the image grid, $\Omega \cong\left\{\left(\mathbf{x}_{i}\right)_{i=1}^{N}\right\}$. In this setting, we can assume $m_{t}$ to be the sum of Dirac measures, where $\alpha_{i}(t)$ is the momentum vector at $\mathbf{x}_{i}$ and time $t$. We use a conjugate gradient routine to perform the minimization of $J$ with respect to $\alpha_{i}(t)$. We summarize steps required in each iteration during the minimization process below:

1. Use the forward Euler method to compute the trajectory based on the flow equation:

$$
\frac{d \phi_{t}\left(\mathbf{x}_{i}\right)}{d t}=\sum_{j=1}^{N} k_{V}\left(\phi_{t}\left(\mathbf{x}_{i}\right), \phi_{t}\left(\mathbf{x}_{j}\right)\right) \alpha_{j}(t) .
$$

2. Compute $\nabla_{\phi_{1}\left(\mathbf{x}_{i}\right)} E$ based on Eq. 16.

3. Solve $\eta_{t}=\left[\eta_{i}(t)\right]_{i=1}^{N}$ in Eq. (9) using the backward Euler integration, where $i$ indices $\mathbf{x}_{i}$, with the initial condition $\eta_{i}(1)=\nabla_{\phi_{1}\left(\mathbf{x}_{i}\right)} E$.

4. Compute the gradient $\nabla J\left(\alpha_{i}(t)\right)=2 \alpha_{i}(t)+\eta_{i}(t)$.

5. Evaluate $J$ when $\alpha_{i}(t)=\alpha_{i}^{\text {old }}(t)-\epsilon \nabla J\left(\alpha_{i}(t)\right)$, where $\epsilon$ is the adaptive step size determined by a golden section search.

\section{Experiments}

In this section, we first illustrate the mapping results of HYDI datasets using LDDMMHYDI and then evaluate the influence of the reorientation on the optimization of the diffeomorphic transformation, which is often neglected in existing DWI-based registration algorithms (e.g., [5]6]). Seven HYDI datasets used in this study consisted of 6 shells corresponding to $b$-values of $0,300,1200,2700,4800$, and $7500 \mathrm{~s} / \mathrm{mm}^{2}$. We refer readers to [15] for more details on the HYDI acquisition. In our experiments, we 
represented HYDI DW signals using the BFOR signal basis with upto the fourth order modified SH bases and upto the sixth order spherical Bessel function. The corresponding BFOR expansion coefficients were used in the LDDMM-HYDI optimization.

Figure 1 shows the LDDMM-HYDI mapping results of three subjects. The last five columns respectively illustrate the geometric shapes of the diffusion signals at five shells of $q$-space in the brain regions with crossing fibers. Red, blue, and green contours respectively represent the shape of the diffusion signals from the subject, template, and deformed template. Visually, the diffusion profile at each shell can be matched well after the mapping. Table 1 lists the squared difference in the diffusion signals of the subjects and the template before and after the LDDMM-HYDI mapping at individual shells in $q$-space, suggesting the significant improvement in the alignment of DWIs after the mapping $(p<0.05)$.

We next evaluated the mapping accuracy of the LDDMM-HYDI algorithms with and without the computation of Term (B) in Eq. (10) during the optimization, where Term (B) seeks the diffeomorphic transformation such that the local diffusion profiles of the template and target HYDIs can be aligned. For this, we first computed the diffusion probability density functions (PDFs) of water molecules, i.e., the ensemble average propagator (EAP), using Fourier transform [12]. Then, we calculated the symmetrized Kullback-Leibler (sKL) divergence between the deformed template and target PDFs [4] in major white matter tracts. The smaller sKL metric indicates the better alignment between the deformed template and target images. The major white matter tracts evaluated in this study include corpus callosum (CC), corticospinal tract (CST), internal capsule (IC), corona radiata (CR), external capsule (EC), cingulum (CG), superior longitudinal fasciculus (SLF), and inferior fronto-occipital fasciculus (IFO). Table 2 lists the values of the mean and standard deviation of the sKL metric for each major white matter tract among six subjects when the LDDMM-HYDI algorithms with and without the Term (B) computation were respectively employed. These results suggest that the LDDMM-HYDI algorithm with the explicit orientation optimization (Term (B) computation) significantly improves the alignment in the major white matter tracts when compared to that without the explicit orientation optimization $(p<0.05)$.

Last, we generated the mDWI atlas by averaging the corresponding BFOR coefficients across seven subjects. For visualizing the neural fiber organization of this atlas, we constructed the EAP image based on the method in [12]. Figure 2] shows the diffusion profiles of this atlas at three layers of the EAP space.

\section{Conclusion}

In this paper, we proposed the LDDMM-HYDI variational problem based on the BFOR signal basis representation of DWIs. We derived the gradient of this variational problem with the explicit computation of the $\mathrm{mDWI}$ reorientation and provided a numeric algorithm without a need of the discretization in $q$-space. Our results showed that the explicit orientation optimization is necessary as it improves the alignment of the diffusion profiles of HYDI datasets.

\section{References}

1. Alexander, D., Pierpaoli, C., Basser, P., Gee, J.: Spatial transformation of diffusion tensor magnetic resonance images. IEEE Trans. on Medical Imaging 20, 1131-1139 (2001) 
Table 1: Evaluation of the LDDMM-HYDI mapping accuracy. The first row lists the squared difference in the diffusion signals of subjects and the template at each shell, while the second row lists that between subjects and the deformed template after the LDDMM-HYDI mapping. The numbers listed are the average and standard deviation values across six subjects.

\begin{tabular}{|c|c|c|c|c|c|}
\hline & $\mathrm{b}=300$ & $\mathrm{~b}=1200$ & $\mathrm{~b}=2700$ & $\mathrm{~b}=4800$ & $\mathrm{~b}=7500$ \\
\hline before LDDMM-HYDI & $7.676(0.800)$ & $5.475(0.318)$ & $2.988(0.135)$ & $2.533(0.071)$ & $2.629(0.109)$ \\
after LDDMM-HYDI & $2.675(0.143)$ & $2.681(0.150)$ & $1.775(0.071)$ & $1.626(0.044)$ & $1.516(0.047)$ \\
\hline
\end{tabular}

A. Atlas

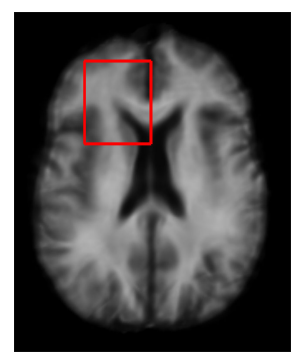

B. $p=5 \mu m$

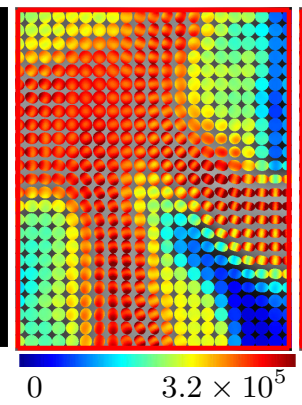

C. $p=10 \mu m$

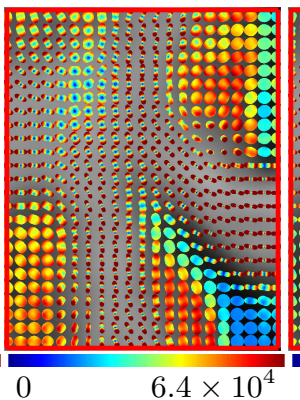

D. $p=15 \mu m$

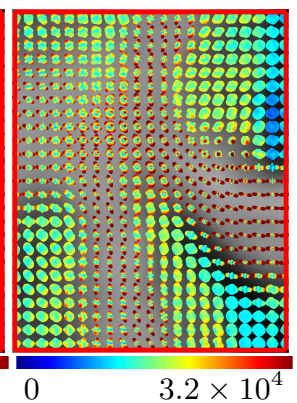

Fig. 2: HYDI atlas in the ensemble average propagator (EAP) space. Panel (A) shows the atlas in terms of zero displacement probability (Po), a scalar image defined in [12] based on EAP. Panels (B-D) respectively illustrate the diffusion profiles of the atlas at three given radii $(p=5,10,15 \mu \mathrm{m})$ in the EAP space. The color indicates the values of EAP.

2. Beg, M.F., Miller, M.I., Trouvé, A., Younes, L.: Computing large deformation metric mappings via geodesic flows of diffeomorphisms. Int. Journal of Computer Vision 61, 139-157 (February 2005)

3. Cetingul, H., Afsari, B., Vidal, R.: An algebraic solution to rotation recovery in hardi from correspondences of orientation distribution functions. In: Biomedical Imaging (ISBI), 2012 9th IEEE International Symposium on. pp. $38-41$ (may 2012)

4. Chiang, M.C., Leow, A., Klunder, A., Dutton, R., Barysheva, M., Rose, S., McMahon, K., de Zubicaray, G., Toga, A., Thompson, P.: Fluid registration of diffusion tensor images using information theory. IEEE Trans. on Medical Imaging 27(4), 442-456 (April 2008)

5. Dhollander, T., Van Hecke, W., Maes, F., Sunaert, S., Suetens, P.: Spatial transformations of high angular resolution diffusion imaging data in Q-space. In: MICCAI CDMRI Workshop. pp. 73-83 (2010)

6. Dhollander, T., Veraart, J., Van Hecke, W., Maes, F., Sunaert, S., Sijbers, J., Suetens, P.: Feasibility and advantages of diffusion weighted imaging atlas construction in q-space. In: Proceedings of the 14th international conference on Medical image computing and computerassisted intervention - Volume Part II. pp. 166-173. MICCAI'11 (2011)

7. Dorst, L.: First order error propagation of the procrustes method for $3 \mathrm{~d}$ attitude estimation. Pattern Analysis and Machine Intelligence, IEEE Transactions on 27(2), 221 -229 (2005)

8. Du, J., Goh, A., Qiu, A.: Diffeomorphic metric mapping of high angular resolution diffusion imaging based on riemannian structure of orientation distribution functions. Medical Imaging, 
Table 2: Table lists the mean and standard deviation values of the symmetrized KullbackLeibler (sKL) divergence of the diffusion probability density functions (PDFs) between the deformed template and target HYDIs in each major white matter tract. The second and third columns show the results obtained from the LDDMM-HYDI with and without the Term (B) computation. * denotes statistical significance indicating that the alignment obtained from the LDDMM-HYDI with the Term (B) computation is better than that obtained from the LDDMM-HYDI without the Term (B) computation at a significance level of 0.05. Abbreviation: CC-corpus callosum; CST-corticospinal tract; ICinternal capsule; CR-corona radiata; EC-external capsule, CG-cingulum, SLF-superior longitudinal fasciculus, and IFO-inferior fronto-occipital fasciculus.

\begin{tabular}{|l|c|c|}
\hline & LDDMM-HYDI with Term (B) & LDDMM-HYDI without Term (B) \\
\hline CST & $0.598(0.265)$ & $0.676(0.305)^{*}$ \\
CC & $0.407(0.180)$ & $0.452(0.202)^{*}$ \\
IC & $0.425(0.189)$ & $0.446(0.200)^{*}$ \\
CR & $0.368(0.163)$ & $0.429(0.191)^{*}$ \\
EC & $0.476(0.212)$ & $0.488(0.218)^{*}$ \\
CG & $0.504(0.223)$ & $0.566(0.252)^{*}$ \\
SLF & $0.409(0.187)$ & $0.527(0.241)^{*}$ \\
IFO & $0.518(0.229)$ & $0.543(0.240)^{*}$ \\
\hline
\end{tabular}

IEEE Transactions on 31(5), $1021-1033$ (2012)

9. Du, J., Younes, L., Qiu, A.: Whole brain diffeomorphic metric mapping via integration of sulcal and gyral curves, cortical surfaces, and images. NeuroImage 56(1), 162 - 173 (2011)

10. Geng, X., Ross, T.J., Gu, H., Shin, W., Zhan, W., Chao, Y.P., Lin, C.P., Schuff, N., Yang, Y.: Diffeomorphic image registration of diffusion mri using spherical harmonics. Medical Imaging, IEEE Transactions on 30(3), 747 -758 (2011)

11. Goh, A., Lenglet, C., Thompson, P., Vidal, R.: Estimating orientation distribution functions with probability density constraints and spatial regularity. In: Medical Image Computing and Computer-Assisted Intervention. pp. 877-885 (2009)

12. Hosseinbor, A.P., Chung, M.K., Wu, Y.C., Alexander, A.L.: Bessel fourier orientation reconstruction (bfor): An analytical diffusion propagator reconstruction for hybrid diffusion imaging and computation of q-space indices. NeuroImage 64(0), 650 - 670 (2013)

13. Hsu, Y.C., Hsu, C.H., Tseng, W.Y.I.: A large deformation diffeomorphic metric mapping solution for diffusion spectrum imaging datasets. NeuroImage 63(2), 818 - 834 (2012)

14. Raffelt, D., Tournier, J.D., Fripp, J., Crozier, S., Connelly, A., Salvado, O.: Symmetric diffeomorphic registration of fibre orientation distributions. NeuroImage 56(3), $1171-1180$ (2011)

15. Wu, Y.C., Alexander, A.L.: Hybrid diffusion imaging. NeuroImage 36(3), 617 - 629 (2007)

16. Yap, P.T., Shen, D.: Spatial transformation of dwi data using non-negative sparse representation. Medical Imaging, IEEE Transactions on 31(11), 2035 -2049 (nov 2012)

17. Yeo, B., Vercauteren, T., Fillard, P., Peyrat, J.M., Pennec, X., Golland, P., Ayache, N., Clatz, O.: Dt-refind: Diffusion tensor registration with exact finite-strain differential. Medical Imaging, IEEE Transactions on 28(12), $1914-1928$ (2009)

18. Zhang, P., Niethammer, M., Shen, D., Yap, P.T.: Large deformation diffeomorphic registration of diffusion-weighted images. In: MICCAI (2012) 


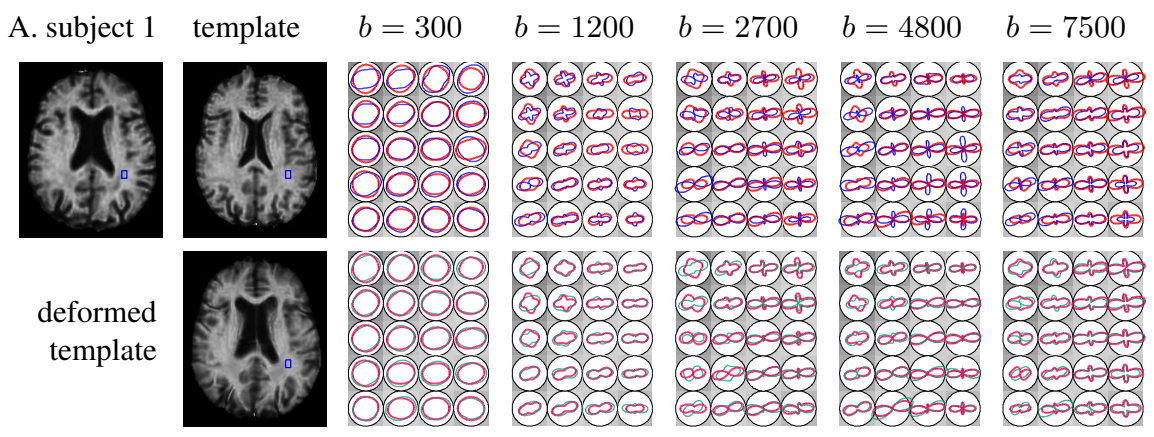

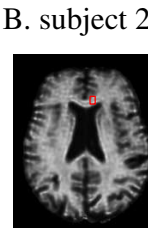

deformed template
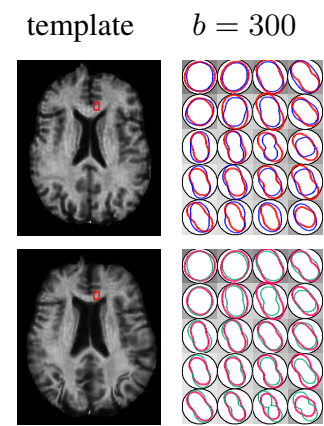

$b=1200$

$b=2700$

$b=4800$

$b=7500$
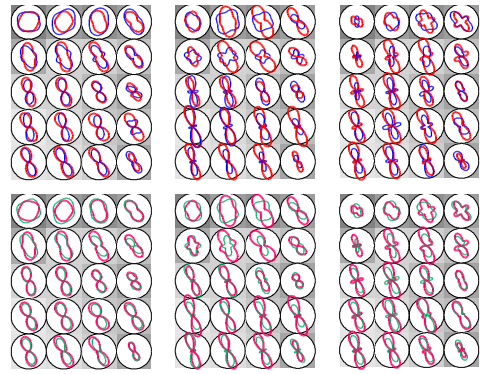

(क) (3)

(4) $8(3)$

7) 888

की 8 (8)

8 \& $8(8)$ \& 8
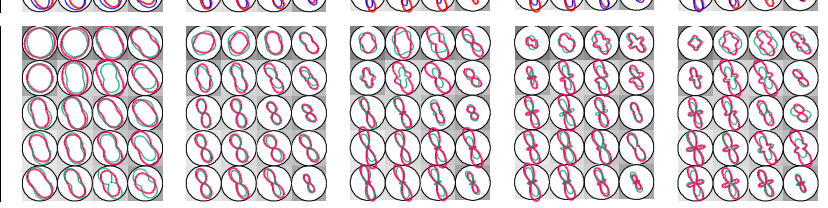

C. subject 3
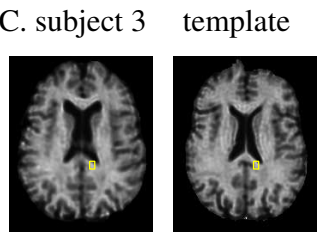

$b=300$
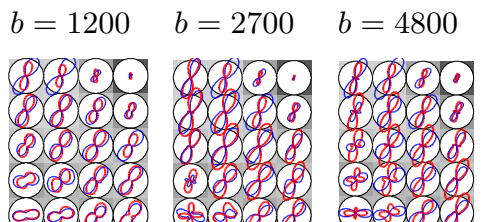

$b=7500$

deformed template
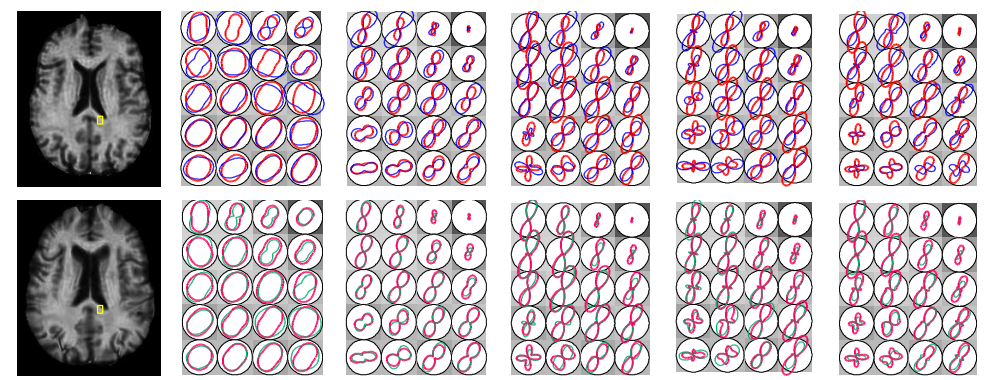

20008

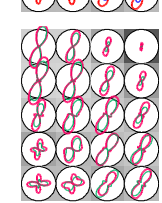

Fig. 1: Illustration of the LDDMM-HYDI mapping results. The first row of panels (AC) illustrates the subject image, template image, the diffusion profiles at individual shells with $b=300,1200,2700,4800$, and $7500 \mathrm{~s} / \mathrm{mm}^{2}$ in $q$-space, respectively. The second row of panels (A-C) illustrates the deformed template image after the LDDMMHYDI mapping, the diffusion profiles at individual shells with $b=300,1200,2700$, 4800 , and $7500 \mathrm{~s} / \mathrm{mm}^{2}$ in $q$-space, respectively. Red, blue, and green contours in the last five columns respectively illustrate the diffusion profiles of the subject, template, and deformed template. The closer the green contour to the red contour, the better the alignment. Note that the profile of diffusion weighted signals is shown in this figure. It is orthogonal to the fiber orientation. 